\title{
Rethinking the service delivery system of psychological interventions in low and middle income countries
}

\author{
L. K. Murray ${ }^{1 *}$ and M. J. D. Jordans $s^{2,3}$
}

\begin{abstract}
Background: Global mental health is a growing field intricately connected to broader health, violence and economic issues. Despite the high prevalence and cost of mental health disorders, an estimated $75 \%$ of those with need in lower resource settings do not receive intervention. Most studies to date have examined the effectiveness of single-disorder mental health treatments - an approach that may be a significant challenge to scale-up and sustainability in lower resource settings.

Main body: This paper presents a brief overview of the scientific progress in global mental health, and suggests consideration of an internal stepped care delivery approach. An internal stepped care model is one idea of a delivery system, utilizing a common elements approach, where the same provider could navigate between different elements based on severity and type of problems of the client. It is distinct from traditional stepped care models in that clients remain with the same provider, rather than relying on referral systems.
\end{abstract}

Conclusion: An internal stepped care delivery system based on a simplified common elements approach could be more efficient, scalable, sustainable, and reduce the loss of clients to referrals in lower resource settings.

Keywords: Global mental health, Implementation science, Common elements approach, Low resource settings, Health systems

\section{Background}

Global mental health research and practice is increasing as evidenced by scientific publications, funders, and growing recognition of its importance in the larger scheme of health and productivity [1-3]. Despite the high prevalence and cost of mental health disorders, an estimated $75 \%$ of those with need do not receive intervention [4-7]. Some of the primary barriers to addressing the mental health intervention gap in low and middle-income countries (LMIC) include limited mental health infrastructure or systems, funding, and scarcity of mental health professionals [8-11].

In the last decade, substantial advances have been made in global mental health (for some reviews see [4, 12-14]). A particular growth includes evidence from randomized controlled trials (RCT) which have demonstrated that

\footnotetext{
* Correspondence: Imurra15@jhu.edu

1 Department of Mental Health, Johns Hopkins Bloomberg School of Public

Health, 624 N. Broadway Street, Baltimore, MD 21205, USA

Full list of author information is available at the end of the article
}

evidence-based treatments (EBT) targeting common mental disorders, primarily based in cognitive behavioral therapy, can be implemented in LMIC with positive clinical outcomes on mental health symptomatology. Most of these studies have evaluated single-disorder-focused interventions (e.g., Interpersonal Psychotherapy for Depression, IPT; Narrative Exposure Therapy for PTSD, NET) [15-23]. Some of these EBTs are recommended in the recent World Health Organization (WHO) Mental Health GAP (mhGAP) Guidelines [24, 25] as frontline interventions.

Another area of advancement is around the effective implementation of evidence-based interventions in LMIC. For example, due to the shortage of highly trained personnel, most of the studies above used a task-shifting approach or the use of non-professionals with limited, if any, formal mental health training as counselor [26]. Studies have also examined the feasibility and cultural modification of EBT [27-32]. 
Despite this scientific evidence and policy support from organizations like WHO, very few organizations or countries have been able to scale-up or sustain EBT that have shown to be effective in LMIC. Some literature exists on potential reasons for the lack of scale-up and sustainability of any of these efficacious interventions $[11,33-35]$. Frequently mentioned challenges are funding, instability, limited trained personnel, logistics (transport, communications), and lack of time and space for delivery of services [10]. More recently, researchers have suggested that the use of single disorder interventions may be problematic and in the long-term not feasible for scale-up [11, 36, 37].

\section{Main text}

To date, much of the research and implementation of mental health interventions in LMIC has followed an approach similar to some high-income countries (HIC). Mental health in HIC is often trained and delivered via "silos" for certain symptoms and/or severities. For example, a designated clinic may treat a particular problem (e.g., a clinic for substance use), and/or have a group of counselors that each has expertise in treating certain disorders. This "silo" model requires a complex system of triage, referrals, and extensive well-trained personnel. It necessitates accurate assessment, followed by referral to either: (a) specific providers depending on the problem, (b) a provider who had trained on and mastered multiple EBTs, or (c) another clinic that specializes in a particular problem or severity (e.g., anxiety disorder clinic, psychiatric clinic).

Many randomized controlled trials in LMIC that have shown strong effectiveness on mental health symptomatology have evaluated interventions that originated from high-income settings (e.g., IPT; Cognitive Processing Therapy, CPT) and that are disorder-specific (i.e., they were designed and tested to treat one primary disorder) [15-23]. Within these trials, a group of lay providers may be trained to treat depression, for example, but would not know how to address trauma, anxiety or other comorbid or common mental health symptoms. The implication of this is that either: (a) the same lay service providers are required to (eventually) be competent in multiple different interventions to serve at a population level, or (b) each provider would have a specialty to only treat one disorder, and therefore many different providers and referral links would be needed.

We suggest that the segregation of services into "silos", either related to symptoms/diagnoses or severity, increases the barriers to scale-up and sustainability in LMIC and hinders the ability to reduce the treatment gap. (See Additional file 1: Figure S1) First, having disorder specific interventions suggests (and requires to a degree) a "fit" into Western diagnostic categories as exemplified in the Diagnostic and Statistic Manual (DSM) and International Classification of Disorders (ICD), which some argue is questionable cross-culturally $[31,38,39]$. Second, comorbidity is the rule - not the exception - along with other problems that can affect the course of intervention (e.g., relationship problems), although these may not meet a diagnostic category. As Weisz (2015) [39] puts it, "stated simply, most EBTs are more narrowly focused, and more linear in design, than the everyday clinical practice they are designed to enhance". Third, with task-shifting being advocated as a strategy to address limited human resources in LMIC $[34,40]$, it is questionable whether this approach is feasible for ultimate scale-up and sustainability since it would require either large numbers of lay providers each focused on a particular mental health problem or that individuals with limited education learn multiple EBT. Both of these would be difficult with task-sharing. Finally, silo'ed care requires options for referral to other providers or settings that are rarely available in many lower resource settings.

\section{A different delivery system conceptualization}

To more effectively reap the benefits of science to practice and scale up of EBTs, a different mental health systems approach may be needed in certain contexts. We suggest consideration of an "internal stepped care model" that allows for the same non-professional service provider (or number of providers) to navigate between different intervention elements based on the severity (i.e. continuous from low to moderate-to-severe) and type of problems (i.e. diverse symptom clusters focused on common mental disorders) of the client.

\section{Based on navigating common elements}

Common elements approaches, also known as transdiagnostic, are increasingly being used, studied and suggested as an alternative way to approach mental health scale up [37, 41-44]. A common elements approach is derived from research showing that most EBTs are actually comprised of many of the same elements or components [42]. In this way, elements are taught (rather than a particular manual), including how to combine them to use for different symptoms and severity levels [43, 44]. Therefore, providers need be trained in only one approach (consisting of common elements and their flexible use), and each provider would be able to treat a range of presenting problems as well as varying severities of common mental health problems depending on the transdiagnostic approach taught (e.g., depression, trauma, anxiety, externalizing symptoms, substance use).

Data on effectiveness of common element approaches is emerging both in high-income countries and LMIC. In the United States and Europe, studies are showing 
positive results across both adult [45-49] and child populations [50]. These approaches are performing at or better than single disorder treatments. However, this work in HIC has been done by mental health professionals. Thus, a significant question about the use of common elements approaches in LMIC is if nonprofessional providers can be trained to select elements based on the needs of a client, both in terms of severity and type of problem and deliver them adequately. The desire to use a common elements approach for scale-up and sustainability would be a mute point if nonprofessional providers with limited education could not learn the multiple elements included in the approach, and know how to put them together for a range of client presentations.

A modular common elements approach was developed specifically for LMIC that was based off current research in the United States with MATCH and the Unified Protocol (UP), $[45,50]$ but with a reduced number of elements and simplified decision rules to account for the training of non-professionals (Common Elements Intervention Approach or CETA) [37]. Briefly, CETA developers utilized distillation research [51] and consultation with developers of multiple evidence-based treatments in an attempt to choose the most frequently used elements, and those that seemed to be the "mechanism of action". Two trials (Iraq and Thailand/Myanmar border) on adult populations that were trauma-affected were completed with CETA - both showing strong effectiveness on symptoms of depression, trauma and anxiety with effect sizes $>1)[52,53]$. In Iraq, CETA performed better than single disorder treatments. One open trial of CETA for youth was completed in Ethiopia with significant results [54]. Although more studies are needed, these studies suggest that: (1) para-professionals are able to learn a simplified common elements approach (inclusive of 9 elements only) with an apprenticeship model of training and ongoing supervision [55], and, (2) that the elements chosen for CETA were collectively as effective or more for comorbid presentations in comparison to single disorder treatments or control conditions.

It is important to note that CETA, MATCH, CBT-E, and UP are examples of common elements approaches that could make an internal stepped care model possible. Although these transdiagnostic treatments utilize CBTbased elements, other elements could be utilized, as long as there was evidence supporting them from rigorous research in accordance with current guidelines on best practices $[4,25]$.

A common elements approach allows for an internal stepped care model (Fig. 1), which is an attempt to address some of the challenges of implementation, reach, scale-up and sustainability of mental health programs in LMIC. In practice, a service provider could be trained in a common elements approach and learn how to put elements together for different common mental health disorders. Upon assessment, this one provider could decide to start with a smaller set of elements due to lower symptoms, and only add elements if there were sustained problems. Alternatively, upon assessment, a provider may note moderate to severe symptoms and choose an order of elements indicated for the presenting

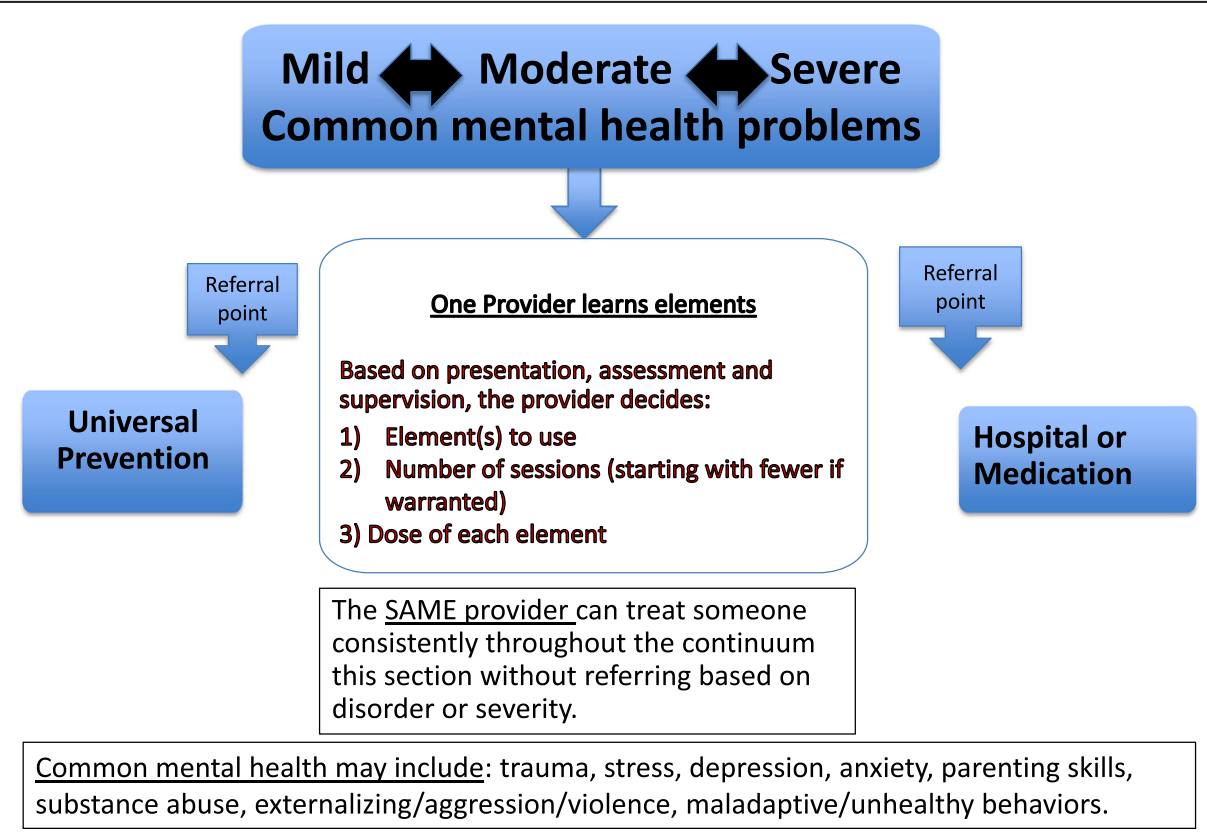

Fig. 1 Internal Stepped Care Approach 
problems based on existing EBT. In either case the service provider would be able to add elements and/or dose of elements based on need and client response. This flexibility, within fidelity to the evidence base, allows the same single provider to address a wide range of problems and severities, and provide only what the client needs based on symptom presentation throughout.

To our knowledge, CETA is the only common elements approach that has been tested in LMIC. Nonprofessionals were successfully trained in the choice patterns discussed above by learning to gleam information from three "data points" throughout treatment: 1) assessment form (client self-report), 2) what the client does and says directly, and 3) consultation with a supervisor. This helps determine what the main problems are of the client. There was not a focus on "diagnoses" as one would in Western psychiatry. Changes could be made to the element choice and dose based on these three information sources throughout treatment. This is one example of how non-professionals could be taught. More research is needed on how well and with how much support non-professional counselors are able to adequately assess the severity and core problems to address in a range of clients.

\section{How is this different from a stepped care approach?}

Stepped care models advocate moving from lowerintensity and least restrictive interventions to higherintensity and more restricted access interventions based on the lack of desired effect of the previous level of care, [56] generally moving from one service provider or organization to the next. One challenge with this type of stepped care approach in lower-resourced settings is the inherent assumption that there is a "next step" if someone does not respond to the first step of intervention. In most LMIC, there are not enough mental health professionals or even lay providers trained in any EBT that could offer services for moderate to severe common mental disorders. In our proposed model, this transfer still happens but within one provider utilizing one approach. The individual could still begin by providing a brief intervention that requires fewer health care resources, but would then be capable of providing ongoing services if the desired intervention benefits were not obtained. The internal stepped care model reduces the need for different groups or levels of provider types, and different specialized settings, which may not be possible in some LMIC settings.

A related challenge with a traditional stepped care model is that it usually includes referral points (from low to high, or across problem area). Every referral point where a client needs to change providers or locations increases the likelihood of them being "lost". Imagine a depressed client who rarely leaves the house, finally making it into a clinic in a low resource area. After a likely long wait and being screened, the lay provider says they do not treat these types of symptoms (e.g., a primary health care worker is insufficiently trained to provide psychological treatments). Although a referral is made, it is quite likely that this client will not make the next referral appointment perhaps due to depressive symptoms, or other reasons such as distance or stigma.

\section{Next steps to consider}

There are numerous research questions that could help determine whether an internal stepped care delivery system is truly beneficial and feasible. First, understanding more about what common elements are needed and used, at what levels of symptomatology, with which symptom clusters, and the doses needed for symptom reduction would further refine the use of common elements approaches. Secondly, although research suggests that clinical decision-making within a common elements approach is possible for para-professionals, $[52,53]$ this model adds variation in symptom presentation and severity beyond these particular studies. Evaluation of the training and supervision needed for an internal stepped care delivery system will be critical. This might include evaluation of key indicators of competency [57] or capacity of trainees in clinical decision making, as well as the amount of resources needed to obtain "adequate" skill levels. Third, common elements approaches have yet to be evaluated in groups - which is a delivery system of interest in many LMIC. Learning how flexibility of element choice and dose fits into group models will need to be studied. Finally, this internal stepped care model is a service delivery framework that addresses some of the known challenges with broader implementation and sustainability of effective mental health interventions. However, it will be important to assess what settings this may or may not work within. Implementation constructs including cost-effectiveness, feasibility, acceptability, and appropriateness will need to be assessed, as well as who provides services and to what degree, to what types and severity of populations.

\section{Conclusions}

The field of global mental health is at an important crossroad where there is now increasing evidence to suggest that some EBTs are effective and feasible in low resource settings - and yet there is limited scale-up or sustainability of these. This means although we have growing information on what works to alleviate mental health suffering, most populations in need are not receiving these services. Thinking through implementation methods is critical. It is clear that the costs of implementing, with fidelity and thus effectiveness, even one EBT are enormous. Consequently it is important to be 
selective when choosing an EBT for implementation that fits within the context and meets the needs of the population. We have suggested that mimicking mental health care delivery approaches that are commonplace in higher resource settings may not be the most sustainable in some LMIC where funding, personnel and infrastructure are lacking. An internal stepped care model is one idea of a delivery system, utilizing a common elements approach, where the same provider could navigate between different elements based on severity and type of problems of the client. This delivery system could be more efficient, scalable, sustainable, and reduce the loss of clients to referrals (existent or non-existent) in LMIC. This certainly does not solve all the challenges found in scaling up global mental health and further research is required to evaluate this strategy in the future.

\section{Additional file}

Additional file 1: Figure S1. Example system flow with "silo'ed" treatments. (PPTX $113 \mathrm{~kb}$ )

\section{Abbreviations}

CBT, cognitive behavioral therapy; CETA, common elements treatment approach; DSM, Diagnostic and Statistic Manual; EBT, evidence-based treatment; ICD, International Classification of Disorders; IPT, Interpersonal Psychotherapy for Depression; LMIC, low and middle-income country; RCT, randomized control trial

\section{Acknowledgements}

The conceptualization of the idea came from experience working with CETA (LKM) funded through the USAID Victims of Torture fund (VOT) Award DFDA-00-08-00308-00. USAIDNOT was not involved in the research or program design or implementation, or in the management or analysis of the data. The ideas were also influenced by work done by TPO Nepal (MJ) within "PRogramme for Improving Mental health carE (PRIME)", funded by UK aid from the UK government (GB-1-201446). However, the views expressed do not necessarily reflect the UK government's official policies.

\section{Funding}

Not applicable.

\section{Availability of data and materials}

Not applicable.

\section{Authors' contributions}

LKM and MJDJ both conceptualized and refined the manuscript idea and wrote the manuscript. Both authors read and approved the final manuscript.

\section{Authors' information}

Not applicable.

\section{Competing interests}

We declare that we are the sole authors of this work and neither of us has a conflict of interest, either financial or otherwise. The contents of the paper have not been published elsewhere, nor being considered for publication. In addition, both authors take responsibility for the integrity of the work as a whole, from inception to published article.

\section{Consent for publication}

Not applicable.

Ethics approval and consent to participate Not applicable.

\section{Author details}

'Department of Mental Health, Johns Hopkins Bloomberg School of Public Health, 624 N. Broadway Street, Baltimore, MD 21205, USA. ${ }^{2}$ Center for Global Mental Health; Institute of Psychiatry, Psychology and Neuroscience, King's

College London, 16 De Crespigny Park, London SE5 8AF, London, UK.

${ }^{3}$ Research and Development Department, War Child Holland, 61G, 1098

Amsterdam, Amsterdam, Netherlands.

Received: 8 January 2016 Accepted: 22 June 2016

Published online: 12 July 2016

\section{References}

1. Collins PY, Insel TR, Chockalingam A, Daar A, Maddox YT. Grand Challenges in Global Mental Health: Integration in Research, Policy, and Practice. PLoS Med. 2013;10. doi:10.1371/journal.pmed.1001434

2. Collins PY, Patel V, Joestl SS, et al. Grand challenges in global mental health. Nature. 2011:475(7354):27-30. doi:10.1038/475027a.

3. The Lancet. A Movement for Global Mental Health is launched. Lancet. 2008;372:1274. doi:10.1016/S0140-6736(08)61528-7.

4. Dua T, Barbui C, Clark N, et al. Evidence-based guidelines for mental, neurological, and substance use disorders in low- and middle-income countries: Summary of WHO recommendations. PLoS Med. 2011;8. doi:10.1371/journal.pmed.1001122.

5. Kohn R, Saxena S, Levav I, Saraceno B. The treatment gap in mental health care. Bull World Health Organ. 2004;82:858-66. doi:S0042-96862004001100011.

6. Saxena S, Thornicroft G, Knapp M, Whiteford H. Resources for mental health: scarcity, inequity, and inefficiency. Lancet. 2007;370:878-89. doi:10.1016/S01406736(07)61239-2.

7. Wang PS, Aguilar-Gaxiola S, Alonso J, et al. Use of mental health services for anxiety, mood, and substance disorders in 17 countries in the WHO world mental health surveys. Lancet. 2007;370(9590):841-50. doi:10.1016/S01406736(07)61414-7.

8. Knapp M, Funk M, Curran C, Prince M, Grigg M, Mcdaid D. Economic barriers to better mental health practice and policy. Health Policy Plan. 2006;21(March):157-70. doi:10.1093/heapol/czl003.

9. Patel V, Flisher AJ, Hetrick S, McGorry P. Mental health of young people: a global public-health challenge. Lancet. 2007:369(9569):1302-13. doi:10.1016/S0140-6736(07)60368-7.

10. Saraceno B, van Ommeren M, Batniji $R$, et al. Barriers to improvement of mental health services in low-income and middle-income countries. Lancet. 2007;370:1164-74. doi:10.1016/S0140-6736(07)61263-X

11. Murray LK, Tol W, Jordans $M$, et al. Dissemination and implementation of evidence based, mental health interventions in post conflict, low resource settings. Intervention. 2014:12:94-112. doi:10.1097/WTF.0000000000000070.

12. van Ginneken N, Tharyan P, Lewin S, et al. Non-specialist health worker interventions for the care of mental, neurological and substance-abuse disorders in low- and middle-income countries. Cochrane Database Syst Rev. 2013:11(11):CD009149. doi:10.1002/14651858.CD009149.pub2.

13. Hanlon C, Luitel NP, Kathree T, et al. Challenges and opportunities for implementing integrated mental health care: A district level situation analysis from five low- and middle-income countries. PLoS One. 2014;9. doi:10.1371/journal.pone.0088437.

14. Patel V, Boyce N, Collins PY, Saxena S, Horton R. A renewed agenda for global mental health. Lancet. 2011;378(9801):1441-2. doi:10.1016/S01406736(11)61385-8.

15. Patel V, Weiss HA, Chowdhary $\mathrm{N}$, et al. Effectiveness of an intervention led by lay health counsellors for depressive and anxiety disorders in primary care in Goa, India (MANAS): A cluster randomised controlled trial. Lancet. 2010;376:2086-95. doi:10.1016/S0140-6736(10)61508-5.

16. Bolton P, Bass J, Neugebauer R, et al. Group interpersonal psychotherapy for depression in rural Uganda: a randomized controlled trial. JAMA. 2003; 289(23):3117-24. doi:10.1001/jama.289.23.3117.

17. Bolton P, Bass J, Betancourt T, et al. Interventions for depression symptoms among adolescent survivors of war and displacement in northern Uganda: a randomized controlled trial. JAMA. 2007;298(5):519-27. doi:10.1001/jama. 298.5.519.

18. Bass JK, Annan J, Mclvor Murray S, et al. Controlled Trial of Psychotherapy for Congolese Survivors of Sexual Violence. N Engl J Med. 2013;368(23): 2182-91. doi:10.1056/NEJMoa1211853.

19. Murray LK, Skavenski S, Kane JC, et al. Effectiveness of trauma-focused cognitive behavioral therapy among trauma-affected children in lusaka, 
zambia: A randomized clinical trial. JAMA Pediatr. 2015;21205:1-9. doi:10.1001/jamapediatrics.2015.0580.

20. Neuner F, Onyut PL, Ertl V, Odenwald M, Schauer E, Elbert T. Treatment of posttraumatic stress disorder by trained lay counselors in an African refugee settlement: a randomized controlled trial. J Consult Clin Psychol. 2008;76(4): 686-94. doi:10.1037/0022-006X.76.4.686.

21. Hensel-Dittmann D, Schauer M, Ruf $M$, et al. Treatment of traumatized victims of war and torture: A randomized controlled comparison of narrative exposure therapy and stress inoculation training. Psychother Psychosom. 2011;80(6):345-52. doi:10.1159/000327253.

22. Ertl V, Pfeiffer A, Schauer E, Elbert T, Neuner F. Community-implemented trauma therapy for former child soldiers in Northern Uganda: a randomized controlled trial. JAMA. 2011;306:503-12. doi:10.1001/jama.2011.1060.

23. Rahman A, Malik A, Sikander S, Roberts C, Creed F. Cognitive behaviour therapy-based intervention by community health workers for mothers with depression and their infants in rural Pakistan: a cluster-randomised controlled trial. Lancet. 2008;372(9642):902-9. doi:10.1016/50140-6736(08)61400-2.

24. WHO. WHO Mental Health Gap Action Programme (mhGAP). 2010.

25. Tol WA, Barbui C, Bisson J, et al. World Health Organization Guidelines for Management of Acute Stress, PTSD, and Bereavement: Key Challenges on the Road Ahead. PLoS Med. 2014;11(12). doi:10.1371/journal.pmed.1001769.

26. World Health Organization. Task Shifting: Rational Redistribution of Tasks among Health Workforce Teams: Global Recommendations and Guidelines:; 2008.

27. Patel V, Chowdhary N, Rahman A, Verdeli H. Improving access to psychological treatments: lessons from developing countries. Behav Res Ther. 2011;49(9):523-8. doi:10.1016/j.brat.2011.06.012.

28. Verdeli H, Clougherty K, Onyango G, et al. Group Interpersonal Psychotherapy for depressed youth in IDP camps in Northern Uganda: adaptation and training. Child Adolesc Psychiatr Clin N Am. 2008;17(3):605-24. doi:10.1016/j. chc.2008.03.002. ix.

29. Murray LK, Dorsey S, Skavenski S, et al. Identification, modification, and implementation of an evidence-based psychotherapy for children in a lowincome country: the use of TF-CBT in Zambia. Int J Ment Health Syst. 2013; 7(1):24. doi:10.1186/1752-4458-7-24.

30. Kaysen D, Lindgren K, Zangana G a. S, Murray L, Bass J, Bolton P. Adaptation of cognitive processing therapy for treatment of torture victims: Experience in Kurdistan, Iraq. Psychol Trauma Theory, Res Pract Policy. 2013;5(2):184-192. doi:10.1037/a0026053.

31. Patel V. Rethinking mental health care : bridging the credibility gap. Intervention. 2014;12(1):15-20.

32. Ventevogel P. Integration of mental health into primary healthcare in lowincome countries: Avoiding medicalization. Int Rev Psychiatry. 2014;26(6): 669-79. doi:10.3109/09540261.2014.966067.

33. Tansella M, Thornicroft G. Implementation science: understanding the translation of evidence into practice. Br J Psychiatry. 2009;195(4):283-5 doi:10.1192/bjp.bp.109.065565.

34. Kakuma R, Minas H, Van Ginneken $\mathrm{N}$, et al. Human resources for mental health care: Current situation and strategies for action. Lancet. 2011;378: 1654-63. doi:10.1016/50140-6736(11)61093-3.

35. Razzouk D, Sharan P, Gallo C, et al. Scarcity and inequity of mental health research resources in low-and-middle income countries: a global survey. Health Policy. 2010:94(3):211-20. doi:10.1016/j.healthpol.2009.09.009.

36. Ventevogel P, Psychological SP. Treatments for Orphans and Vulnerable Children Affected by Traumatic Events and Chronic Adversity in subSaharan Africa. JAMA Pediatr. 2015;314(5):511-2.

37. Murray LK, Dorsey S, Haroz E, et al. A common elements treatment approach for adult mental health problems in low- and middle-income countries. Cogn Behav Pract. 2014;21:111-23. doi:10.1016/..cbpra.2013.06.005.

38. Jacob KS, Patel V. Classification of mental disorders: a global mental health perspective. Lancet. 2014;383(9926):1433-5. doi:10.1016/S0140-6736(13)62382-X.

39. Weisz JR, Krumholz LS, Santucci L, Thomassin K, Ng MY. Shrinking the Gap Between Research and Practice: Tailoring and Testing Youth Psychotherapies in Clinical Care Contexts. Annu Rev Clin Psychol. 2015;11(1): 139-63. doi:10.1146/annurev-clinpsy-032814-112820.

40. Mendenhall E, De Silva MJ, Hanlon C, et al. Acceptability and feasibility of using non-specialist health workers to deliver mental health care: Stakeholder perceptions from the PRIME district sites in Ethiopia, India, Nepal, South Africa, and Uganda. Soc Sci Med. 2014;118C:33-42. doi:10.1016/j.socscimed.2014.07.057.
41. Jordans MJD, Tol WA, Komproe IH. Mental health interventions for children in adversity: Pilot-testing a research strategy for treatment selection in lowincome settings. Soc Sci Med. 2011;73(3):456-66. doi:10.1016/j.socscimed. 2011.06.004.

42. Chorpita BF, Daleiden EL, Weisz JR. Identifying and selecting the common elements of evidence based interventions: A distillation and matching model. Ment Health Serv Res. 2005;7(1):5-20. doi:10.1007/s11020-005-1962-6.

43. McHugh RK, Murray HW, Barlow DH. Balancing fidelity and adaptation in the dissemination of empirically-supported treatments: The promise of transdiagnostic interventions. Behav Res Ther. 2009;47(11):946-53. doi:10.1016/j.brat.2009.07.005.

44. Mansell W, Harvey A, Watkins E, Shafran R. Conceptual Foundations of the Transdiagnostic Approach to CBT. J Cogn Psychother. 2009;23(1):6-19. doi:10.1891/0889-8391.23.1.6

45. Farchione TJ, Fairholme CP, Ellard KK, et al. Unified Protocol for Transdiagnostic Treatment of Emotional Disorders: A Randomized Controlled Trial. Behav Ther. 2012:43(3):666-78. doi:10.1016/j.beth.2012.01.001.

46. Fairburn CG, Bailey-Straebler S, Basden S, et al. A transdiagnostic comparison of enhanced cognitive behaviour therapy (CBT-E) and interpersonal psychotherapy in the treatment of eating disorders. Behav Res Ther. 2015;70:64-71. doi:10.1016/j.brat.2015.04.010.

47. Norton PJ, Barrera TL. Transdiagnostic versus diagnosis-specific CBT for anxiety disorders: A preliminary randomized controlled noninferiority trial. Depress Anxiety. 2012;29(10):874-82. doi:10.1002/da.21974.

48. Norton PJ, Barrera TL, Mathew AR, et al. Effect of transdiagnostic cbt for anxiety disorders on comorbid diagnoses. Depress Anxiety. 2013;30(2):168-73. doi:10.1002/da.22018.

49. Dear BF, Staples LG, Terides MD, et al. Transdiagnostic versus disorder-specific and clinician-guided versus self-guided internet-delivered treatment for generalized anxiety disorder and comorbid disorders: A randomized controlled trial. J Anxiety Disord. 2015;36:63-77. doi:10.1016/j.janxdis.2015.09.003.

50. Weisz JR, Chorpita BF, Palinkas $L A$, et al. Testing standard and modular designs for psychotherapy treating depression, anxiety, and conduct problems in youth: a randomized effectiveness trial. Arch Gen Psychiatry. 2012;69(3):274-82. doi:10.1001/archgenpsychiatry.2011.147.

51. Chorpita BF, Daleiden EL. Mapping evidence-based treatments for children and adolescents: application of the distillation and matching model to 615 treatments from 322 randomized trials. J Consult Clin Psychol. 2009;77(3): 566-79. doi:10.1037/a0014565.

52. Bolton P, Lee C, Haroz EE, et al. A Transdiagnostic Community-Based Mental Health Treatment for Comorbid Disorders: Development and Outcomes of a Randomized Controlled Trial among Burmese Refugees in Thailand. PLoS Med. 2014;11(11). doi:10.1371/journal.pmed.1001757.

53. Weiss WM, Murray LK, Zangana GAS, et al. Community-based mental health treatments for survivors of torture and militant attacks in Southern Iraq: a randomized control trial. BMC Psychiatry. 2015;15(1):249. doi:10.1186/s12888015-0622-7.

54. Murray LK, Hall B, Dorsey S, Ugeuto A, Puffer E, Ismael A, Bass J, Erikson A, Lucid L, harrison J, Akiba C BP. An open trial of a common elements approach to address children's mental health in Somali refugee camps. Submiss.

55. Murray LK, Dorsey S, Bolton P, et al. Building capacity in mental health interventions in low resource countries: an apprenticeship model for training local providers. Int J Ment Health Syst. 2011;5(1). doi:10.1186/17524458-5-30.

56. Bower P, Gilbody S. Stepped care in psychological therapies: Access, effectiveness and efficiency. Narrative literature review. Br J Psychiatry. 2005; 186(JAN):11-7. doi:10.1192/bjp.186.1.11

57. Kohrt BA, Jordans MJD, Rai S, et al. Therapist competence in global mental health: Development of the ENhancing Assessment of Common Therapeutic factors (ENACT) rating scale. Behav Res Ther. 2015;69:11-21. doi:10.1016/j.brat.2015.03.009 